\title{
Thoughts Toward a Theory of Natural Selection: The Importance of Microbial Experimental Evolution
}

\author{
Daniel Dykhuizen \\ Department of Ecology and Evolution, Stony Brook University, Stony Brook, New York 11794 \\ Correspondence: Daniel.dykhuizen@stonybrook.edu
}

\begin{abstract}
Natural selection should no longer be thought of simply as a primitive (magical) concept that can be used to support all kinds of evolutionary theorizing. We need to develop causal theories of natural selection; how it arises. Because the factors contributing to the creation of natural selection are expected to be complex and intertwined, theories explaining the causes of natural selection can only be developed through the experimental method. Microbial experimental evolution provides many benefits that using other organisms does not. Microorganisms are small, so millions can be housed in a test tube; they have short generation times, so evolution over hundreds of generations can be easily studied; they can grow in chemically defined media, so the environment can be precisely defined; and they can be frozen, so the fitness of strains or populations can be directly compared across time. Microbial evolution experiments can be divided into two types. The first is to measure the selection coefficient of two known strains over the first 50 or so generations, before advantageous mutations rise to high frequency. This type of experiment can be used to directly test hypotheses. The second is to allow microbial cultures to evolve over many hundreds or thousands of generations and follow the genetic changes, to infer what phenotypes are selected. In the last section of this article, I propose that selection coefficients are not constant, but change as the population becomes fitter, introducing the idea of the selection space.
\end{abstract}

$T^{\mathrm{h}}$ his article is about natural selection. For many years, I have asked my undergraduate students to memorize this definition of natural selection: Natural selection is the differential reproduction and survival of different phenotypes when, at least, part of the differences in phenotypes is caused by differences in genotype. This can also be expressed as differential growth rates of subpopulations when the subpopulations are distinguished by genetic differences. When expressed as differences in the growth rates in terms of the Malthusian growth parameter, $m$, then natural selection is the difference in birth rates minus the difference in death rates.

From demography, we know that birth rates are very variable depending on the environment. In humans in the United States, the birth rate dropped during the economic depression of the 1930s, rose after World War II to produce a baby boom, and dropped afterward. Worldwide, birth rates drop with the provision of

Editor: Howard Ochman

Additional Perspectives on Microbial Evolution available at www.cshperspectives.org

Copyright (C) 2016 Cold Spring Harbor Laboratory Press; all rights reserved; doi: 10.1101/cshperspect.a018044

Cite this article as Cold Spring Harb Perspect Biol 2016;8:a018044 
government-provided old-age assistance, also with the increasing survival of children previously born. Thus, birth rates are very sensitive to many environmental conditions. Likewise for death rates. We have long known that starvation, disease, war, and fratricide will increase the death rate, often dramatically. There has been a drop in death rates since 1750 as transportation and social organization improved, preventing starvation in local areas as the crops failed. The 1918 flu spiked the death rate and disease could again raise the death rate dramatically. The black plague is famous for wiping out a third to half of some European populations and changing social conditions. Today, high fructose sweetener is blamed for increasing the death rate among lower class Americans. Thus, the environment changes birth and death rates, sometimes dramatically, sometimes very subtly.

Turning back to natural selection, natural selection is the difference between two subpopulations, defined by a genetic difference in their birth and death rates weighted by the effects of all environments experienced by these subpopulations over the time period of the observation. Will natural selection be even more complex than population demography or will it be simpler? It could be much more complicated because the response of the birth and death rates of the two subpopulations in the different environments could be different, giving different norms of reaction. Also, the epistasis and dom- inance could make the reactions of various individuals within each subpopulation to the changing environment very different. Or it could be much simpler when the genetic difference gives different effects only in one environment. For example, continued synthesis of the lactase gene is selected in human populations that ingest lactose as adults.

This complexity embedded in the concept of natural selection has been known for a long time. In population genetics, it is assumed that one can estimate an average selection coefficient over all the environments experienced by the population in a set period without needing to specify the environments or their effect on birth and death rates. This selection coefficient is then used to project gene frequency change over time. Because population genetics is interested in the effects or consequences of natural selection, not the causes, it is satisfactory to treat natural selection as a constant without understanding the causes of natural selection. Unfortunately, this simplification has led to a caricature of natural selection as a constant, given a genetic difference.

The model of natural selection that I currently use is given in Figure 1. Here, the definition of natural selection as I gave to my students is an expanded definition because phenotypes are generated by genotypes in an environment (the epigenetic environment) and natural selection is generated by differences in phenotypes

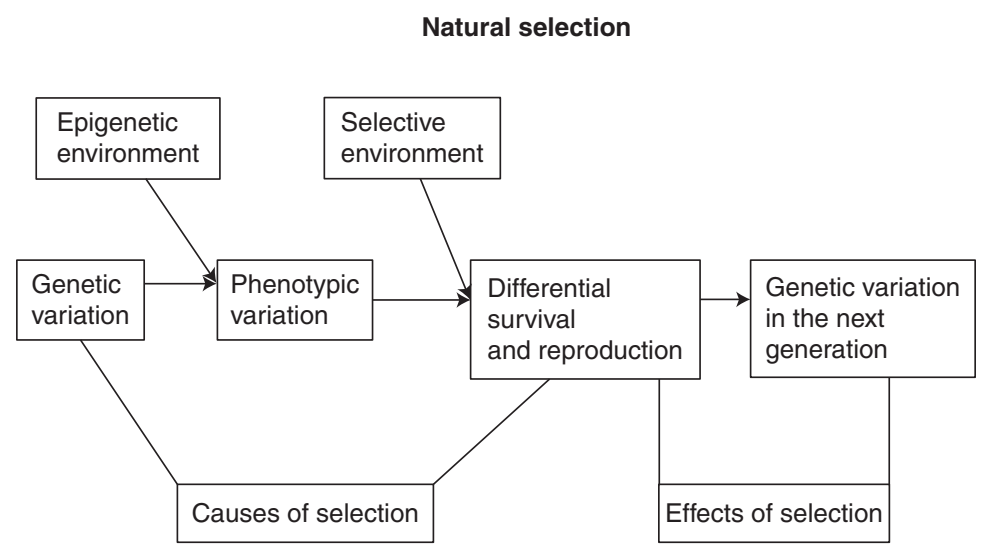

Figure 1. The current model of natural selection indicating the complexity of its causes and distinguishing causes from effects. Population genetics studies only the effects of natural selection. 
in an environment (the selective environment). The interaction of genetic variation, epigenetic environment, phenotypic variation, and the selective environment generate natural selection. These are the "causes" of natural selection. The "effects" of natural selection produce changes in allele frequencies giving rise to adaptive evolution. I believe that the most important function of experimental evolution will be to figure out the causal rules or laws of natural selection. I have previously made the analogy of natural selection evolution with force in physics (Dykhuizen 1995). Newton described the effects of force; the understanding of the causes of force were performed over the next 300 years leading to an understanding of electromagnetism, thermodynamics, atomic energy, etc. This understanding has led to most of the practical applications from physics. Hopefully, the same can be performed for natural selection. But, as the causes of force were much stranger than expected, the causes of natural selection will be stranger than we now imagine. Only by doing experiments will we be forced to accept whatever strangeness there is in natural selection.

\section{EXPERIMENTAL EVOLUTION}

An experiment is the controlled testing of a hypothesis, using an orderly and well-described procedure. An experiment should be performed in such a way that any other scientist anywhere in the world can repeat the experiment and, by using the same logical analysis of the results, obtain the same conclusion. Usually, experiments are designed to provide insight into cause and effect, but also can be used to describe an array of possible outcomes from an initial condition. When possible, experimenters try to design controls such that unknown or unexpected causes, such as the phases of the moon, can be ruled out as a cause of the results. Repeatability is the hallmark of the experimental method.

The experimental method requires that the investigator create the conditions under which the experiment is performed in such a way that the experiment can be repeated. This is usually performed in a laboratory in which conditions are not natural—constant temperature, regular light intensity, defined media without the mix of enzyme inhibitors, toxins, tannins, and other nonmetabolizable compounds found in nature. Also, generally, there are no predators or parasites in the laboratory along with the organisms studied. In such unnatural conditions, what can you learn about the evolution in the world out there? The traditional answer is: "Not much," but I will try to convince you that in one respect this answer is incorrect. It is correct in that experimental evolution will tell us little about the evolution of a particular phenotype of a species in nature. But it is incorrect in that experimental evolution can be used to understand fundamental patterns in nature. I would go further and say that we will never understand ecology and evolution without doing experiments.

It has been argued that experiments in physiology, biochemistry, and genetics can be performed in the laboratory because the physiological, biochemical, or genetic system is not changed when pulled out of its natural environment. The DNA is the same whether it is in an organism in nature or in the laboratory. But, one cannot bring a piece of the environment into the laboratory and have it remain the same. The environment is too complex and too much of a network of cause and effect to do this. If you take a sample of a pond in a jar, the sample in that jar very quickly changes such that the dynamics in the jar become different than the dynamics in a similar piece of the lake. The conclusion is that the environment cannot be moved into the laboratory the way an organism can.

While true, this is an incorrect way to look at the problem. The environment in the laboratory does not need to be natural. When we measure the properties of enzymes, we measure the $V_{\max }$ and $k_{m}$ in dilute salt solutions with excess substrate, an enzyme environment very different from its environment in a cell. The environment of an enzyme in a cell is almost a gel of other compounds, not a dilute salt solution, and contains little substrate, rather than excess. But, yet, these parameters of $V_{\max }$ and $k_{m}$ can predict the fitness ranking of allozymes in their natural environment of the cell (Dykhuizen and Dean 1990). Thus, we believe that characterizing enzymes in dilute salt solutions with excess sub- 
strate gives us real information about enzyme function in its natural state. Likewise, we can study organisms in very unnatural conditions and learn about the process of evolution.

\section{THE FIELD OF MICROBIAL EXPERIMENTAL EVOLUTION}

Experimental evolution as a field of study within evolutionary biology did not really start to develop until the 1970s for a number of reasons. First, Darwin proposed that evolutionary change was very slow and gradual. This was the intellectual default position among evolutionary biologists until very recently. Selection coefficients must be very small and they would be difficult to observe, so it was assumed that meaningful results were likely to take too long, even for laboratory experiments. Laboratory experiments were discouraged at that time also because of the problem mentioned above. If the environment is not natural, will the selection be natural?

Natural selection as studied in experimental evolution is different from artificial selection. In artificial selection, the scientist picks the traits to be selected and breeds from those individuals that best exemplify these traits. In experimental evolution, the scientist creates the environment in which the natural selection happens. Unlike Garland and Rose (2010), I do not consider artificial selection experiments as part of experimental evolution, nor do I consider domestication a part of experimental evolution. However, the line between artificial selection and natural selection can be fuzzy when the investigator sets an environmental condition that is there simply to select a certain defined phenotype (Ratcliff et al. 2012).

There was a small field that has remained distinct from experimental evolution (summarized in Mortlock 1984), which could be revisited, expanded, and become incorporated into experimental evolution. The defining observation of this field is that sometimes a few colonies will grow on a solid media with a novel carbon source in which the majority of the cells do not grow, and this ability is inherited. The genetic changes permitting growth were determined. Usually, one or more enzymes were recruited to establish a new metabolic pathway. These enzymes had some cross reactivity such that they were able to metabolize, very inefficiently, the new compounds. The genetic changes giving growth were mutations making some of these enzymes constitutive and were amplifications of the enzyme genes, such that the cells produced many inefficient enzymes. However, because the only criterion used in these experiments was growth or nongrowth on plates, there was no way to further evolve these cells to see how the new pathway would evolve. This could be performed now using techniques not available in the early 1980s.

Another reason that microbial experimental evolution took a long time to establish was that microbiology was not part of the modern synthesis of 1939-1959. None of the founders of the modern synthesis had expertise in microbiology and so excluded microbiology. Typical of that attitude is the statement by Dobzhansky (1951) in a chapter on species as natural units: "The subdivision of the mass of clones into species Escherichia coli, Salmonella typhosa, and Salmonella enteritidis is purely a matter of taste; one might just as well regard them all as a single species" (p. 274). The hidden assumption was that, because bacteria do not have defined species, they were of no interest in the study of the origin of species by evolution. The microbiologists agreed. There were too few stable morphological markers like Gram-positive and the spiral-shape to establish a phylogeny of bacteria and define species. Microbiologists gave up trying to include microorganisms into the evolutionary-based organismal biology as represented by the modern synthesis (Woese 1994). Instead, microbiology aligned itself with immunology, where only the surface of the bacterial cell was important, and with the rapidly growing field of molecular biology. Molecular biology, established by geneticists and physicists who were looking for the universal principles, was ahistorical. This would not have been a serious problem except that many molecular biologists of the era (1970-2000) looked down on the organismal biologists as old-fashioned and thought that organismal biologists and taxonomists could be eliminated from biology depart- 
ments without loss. During this period, many biology departments split into departments of molecular and cell biology and departments of organismal biology or departments of ecology and evolution. This division looked as if it would grow until the two sections of biology could not even communicate. Ironically, the crowning achievement of molecular biology, genome sequencing, has forced these two sections of biology back together.

A few of us (Barry Hall, Julian Adams, Bruce Levin, and myself) started doing experimental evolution in the 1970s, using microorganisms. Before that time, there had been individual laboratories that had performed an experiment or two in microbial evolution (e.g., Atwood et al. 1951; Zamenhof and Eichhorn 1967). But, we were doing it as the major activity in our laboratories. In 1983, we started the Microbial Population Biology Gordon Conference, which still meets every other year. This meeting has served as an institutional focus for microbial experimental evolution and as a way for evolutionary biologists and molecular biologists to discuss common problems. Now, microbial experimental evolution has a meeting of its own. The first ASM conference on experimental microbial evolution was held in July 2014 and was such a success that future meetings will be held. Microbial experimental evolution is now a recognized and growing field of study. But, I want to finish this article saying that microbial experimental evolution can be used to study natural selection, showing that it can be performed and pointing out anomalies.

\section{THE CAUSES OF NATURAL SELECTION CAN BE UNDERSTOOD: BOTTOM-UP ANALYSIS OF THE LACTOSE SYSTEM}

In this section, I will show that natural selection can be understood to the extent that true predictions can be made about the coefficients of natural selection.

\section{The Lactose Operon}

The lac operon (Fig. 2A) has long served as a model of prokaryotic gene expression (Jacob and Monod 1961; Miller and Reznikoff 1978). In the absence of inducer, the lacI encoded repressor binds to the lac operator, physically blocking transcription. When inducer binds to the repressor, it produces an inactive complex with low affinity for the operator. With the blockage removed, transcription proceeds to synthesize a polycistronic lacZYA mRNA. Isopropyl-thio- $\beta$-galactoside (IPTG) is an effective inducer of the lactose operon that cannot be metabolized by $\beta$-galactosidase and can be used to induce regulated lac operons.

\section{The Lactose Pathway}

The lactose pathway (Fig. 2B) has three steps: passive diffusion of lactose through the porin pores of the outer cell wall into the periplasm, proton coupled import of lactose by the lacY encoded permease into the cytoplasm, and irreversible hydrolysis by the lacZ-encoded $\beta$-galactosidase. The products, glucose and galactose, enter central metabolism directly. The lac operon thus encodes only two of the three steps in the pathway. The lactose system is a simple linear pathway in which the metabolic flux can be precisely modeled following the approach taken by Kacser and Burns (1973) (see below). Because the system can be precisely modeled, this system can be used to make precise predictions about the direction and intensity of selection, predictions that can be tested experimentally.

\section{The Chemostat Environment}

Natural selection happens in an environment. In these experiments, the environment used is a phosphate buffered inorganic minimal media with sugar for both carbon and energy as the sole limiting resource in a $30 \mathrm{~mL}$ Kubitschek style chemostat (Dykhuizen 1993). Sterile hydrated air is pumped into the base of the chemostat to mix and aerate the culture. The generation time is set at $2 \mathrm{~h}$, the temperature at $37^{\circ} \mathrm{C}$ and cell density, which is determined by the concentration of sugar in the fresh medium entering the chemostat, at $7.5 \times 10^{7}$ cells $/ \mathrm{mL}$. Thus, cells compete for a single limiting sugar or sugars and those cells that can 
D. Dykhuizen

A

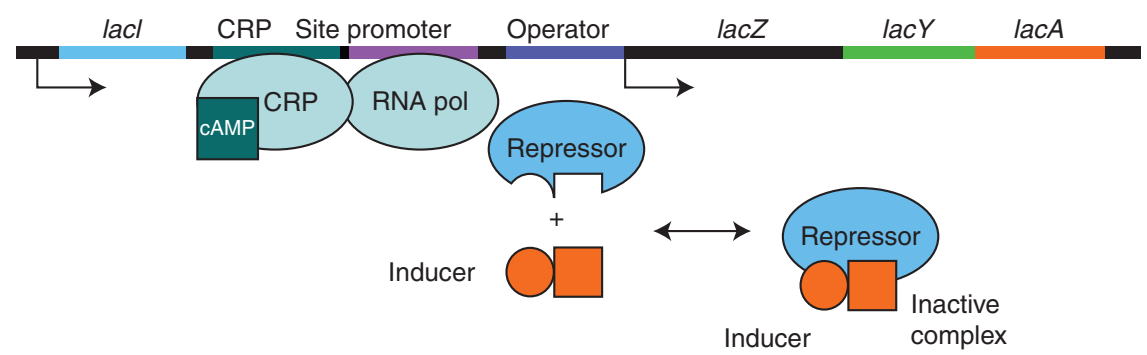

B

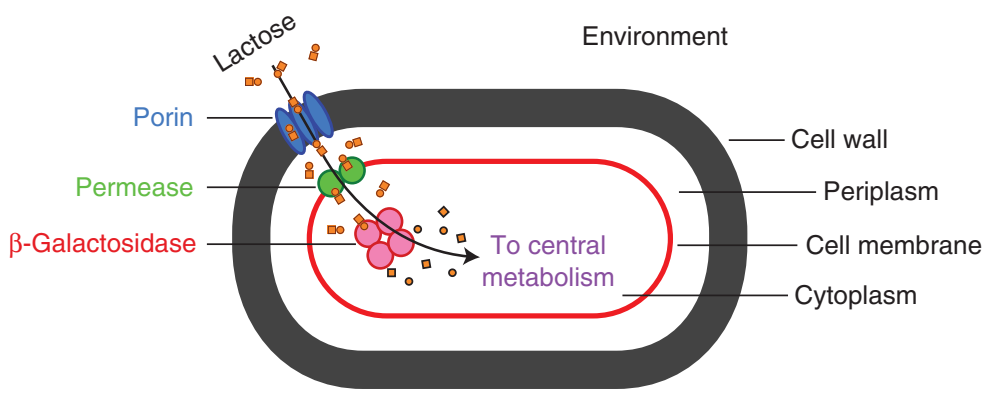

Figure 2. System for the metabolism of lactose. $(A)$ A cartoon of the lactose operon. $(B)$ A cartoon of the lactose pathway. In $A$, lacI is the gene that codes for the repressor of the lactose operon; lac $Z$ codes for the $\beta$-galactosidase; $l a c Y$ codes for the permease; and lacA codes for the transacetalyse that inactivates competitive inhibitors of the $\beta$-galactosidase. In defined media without inhibitors, the transacetalyse is not important and can be neglected. In $B$, the porin, coded for $o m p F$, is not part of the lactose operon.

acquire more of the limiting nutrient, having a higher nutrient flux, will grow faster and be selected for. The concentration of the limiting nutrient in the media is the selective environment. IPTG is added to the media so that the epigenetic environment is constant for any concentration of lactose in the media and the phenotype of the bacteria remains the same across all experiments.

\section{From Fitness to Flux}

As Monod (1942) first showed, growth rate $(\mu)$ is proportional to the concentration of a growth-limiting nutrient. This implies

$$
\mu=Y J
$$

where $Y$ is the yield coefficient (the number of "moles" of cells produced per mole of lactose consumed) and $J$ is the flux of lactose into the cell (the number of "moles" of lactose consumed per "mole" of cells per second). Defining relative fitness $\left(w_{w t}^{i}\right)$ as the growth rate of strain $i$ relative to wild type,

$$
\begin{aligned}
w_{w t}^{i} & =\mu_{i} / \mu_{w t}=Y_{i} J_{i} / Y_{w t} J_{w t}=J_{i} / J_{w t} \\
& =J_{w t}^{i} .
\end{aligned}
$$

Mutations that affect flux do not impact yield and the yield coefficients cancel. During starvation on lactose, we expect relative fitness to be determined by relative flux $\left(w_{w t}^{i}=j_{w t}^{i}\right)$.

\section{From Flux to Enzyme Activities}

Following Kacser and Burns (1973, 1981), the flux of lactose through the pathway $(J$, the in 
vivo rate of lactose hydrolysis) is described by,

$$
J=\frac{L}{\frac{1}{D^{p o r}}+\left(\frac{K_{\max }^{\text {perm }}}{V_{\max }^{\text {pra }}}\right) / K_{e q}^{\text {por }}+\left(\frac{K_{\max }^{\beta-g a l}}{V_{\max }^{\beta-g a l}}\right) / K_{e q}^{\text {por }} \cdot K_{e q}^{\text {perm }}},
$$

where $L$ is the concentration of lactose in the environment (Dean 1989), $D^{\text {por }}$ is the pseudo firstorder rate constant of lactose diffusion through the porin pores, the $K_{m}$ 's are the Michaelis constants of the permease (perm) and $\beta$-galactosidase ( $\beta$-gal) enzymes and the $V_{\text {max }}$ 's are the maximum velocities (calculated on a per cell basis) at each step. $K_{e q}^{\text {por }}=1$ is the equilibrium constant associated with the passive diffusion lactose through the porins. $K_{e q}^{p e r m}=444$ is the apparent equilibrium constant associated with $\mathrm{H}^{+}$symport of lactose by the permease. Lactose hydrolysis is essentially irreversible $\left(K_{e q}^{\beta-g a l} \rightarrow \infty\right)$ and downstream enzymes do not appear in this equation.

\section{The Biochemical Basis of Fitness}

Fitness is the ratio of growth rates that are determined by relative flux of the limiting lactose. Flux is described in terms of the enzyme activities of the enzymes in the pathway. Then

$$
\begin{aligned}
& w(i / w t)=j(i / w t) \\
& =\frac{\frac{1}{D^{p o r}}+\left(\frac{K_{m w t}^{p e r m}}{V_{\max w t}^{p e r m}}\right) / K_{e q}^{p o r}+\left(\frac{K_{m w t}^{\beta-g a l}}{V_{\max w t}^{\beta-g a l}}\right) /\left(K_{e q}^{p o r} \cdot K_{e q}^{p e r m}\right)}{\frac{1}{D^{p o r}}+\left(\frac{K_{m i}^{p e r m}}{V_{\max i}^{p e r m}}\right) / K_{e q}^{p o r}+\left(\frac{K_{m i}^{\beta-g a l}}{V_{\max i}^{\beta-g a l}}\right) /\left(K_{e q}^{p o r} \cdot K_{e q}^{p e r m}\right)}
\end{aligned}
$$

Understand that there are no unknowns in Equation 4-everything has been measured. The straight line in Figure 3 is the theoretical expectation, not a fit to the data. Understand that Equation 4 is not an ad hoc guess at some plausible relationship, but a statement of mechanism derived $\mathrm{ab}$ initio. In this experimental system, natural selection is reduced to biochemistry, and biochemistry can be used to predict the direction and intensity of natural selection precisely.



Figure 3. Fitness-flux relationship. The line is the theoretical line transferring flux into fitness. The black circle is the wild-type K12 operon. The black square is for an inactive $\beta$-galactosidase. The open circles are for various lac operons from various wild strains and from various mutations of genes in the lac pathway that change flux. The open triangles represent various mutations of ebg gene (Hall 1984).

Fitness Is a Hyperbolic Function of Enzyme Activity

Equation 4 can be rewritten in the form:

$$
W_{i}=\frac{1}{C_{D} / D_{i}+C_{P} / D_{i}+C_{\beta} / D_{\beta i}},
$$

where $D_{i}, P_{i}$, and $\beta_{I}$ are the activities of the porins, permease, and $\beta$-galactosidase in strain $i$ when normalized to those of wild-type (E. coli $\mathrm{K} 12)$, and $C_{D}=0.840 \pm 0.028, C_{P}=0.156 \pm$ 0.012 , and $C_{\beta}=0.004 \pm 0.0004$ are the control coefficients of the wild-type E. coli K12 (Kacser and Burns 1981; Dean 1989). The control coefficients are tangents to the curves of TD2 at $x=$ $100, y=1$ (Fig. 4). They measure the degree to which each step limits the flux (fitness). A maximum value of 1 corresponds to a classic "ratelimiting" metabolic step and the minimum of 0 corresponds to "excess" enzyme. The wildtype $\beta$-galactosidase lies on a fitness plateau. Substantial changes in $\beta$-galactosidase activity have little effect on fitness even though the enzyme contributes to a flux under intense direc- 


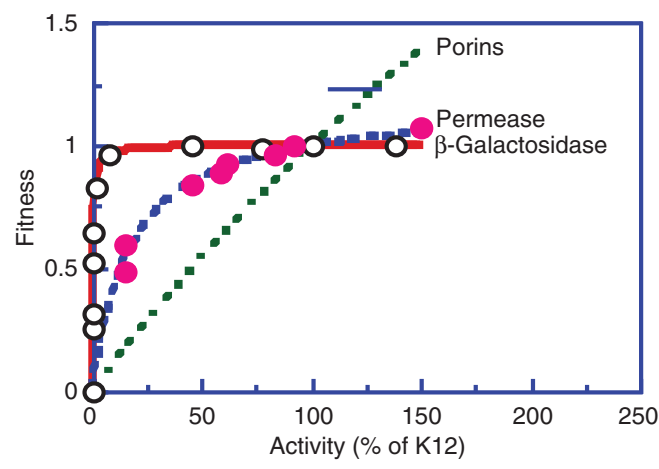

Figure 4. Fitness-enzyme activity relationship. The data used to estimate the control coefficients of the lactose pathway when growing in limiting lactose. The control coefficient for $\beta$-galactosidase is estimated to be 0.004 and the control coefficient for lactose permease is estimated to be 0.156 . The porin control coefficient $(0.840)$ is estimated using the summation theorem, which states that the control coefficients of a linear pathway must add to one (Kacser and Burns 1973).

tional selection. The take-home message is clear. Functional variation is necessary, but not sufficient, for selection to act.

The porin is nearly rate limiting and is expected to the target of selection. Regulatory mutations increasing OmpF porin expression have been isolated from chemostats (Zhang and Ferenci 1999). Also isolated from long-term chemostat cultures are small intragenic deletions and point mutations that remove a constriction in OmpF, increasing the channel diameter and so reducing resistance to lactose diffusion (Zhang and Ferenci 1999). Hence, an understanding of the molecular basis of fitness enabled epistatic shifts in the target of selection, as well as the phenotypes of the adaptive mutations, to be predicted $\mathrm{ab}$ initio.

\section{Other $\beta$-galactosides}

Lactose (galactose-glucose) is not the only $\beta$ galactoside that can be metabolized by the lactose pathway. Some of the other ones are lactulose (galactose-fructose), gal-ara (galactose-arabinose) and methylgalactose (galactose-methyl). The control coefficients of these other $\beta$-galactosides are different from the control coefficients of lactose. Thus, it is possible for one lactose operon to be fitter that another lactose operon (from a different strain, transduced into a K12 background) on one galactoside but less fit on another galactoside. This has been found. Strain TD2 (lactose operon and background from E. coli K12) is fitter than strain TD10 (lactose operon from ECOR16 [Ochman and Selander 1984] and background from K12) on lactulose but less fit on methylgalactose (Dean 1995). Frequency-dependent selection should maintain both strains when growing in a chemostat in which the growth-limiting substrate is a mixture of these two galactosides.

\section{Resource-Based Competition Theory}

Monod (1942, 1950) introduced a resourcebased competition theory to describe microbial growth in chemostats relating limiting resource concentration to growth rate. This was extended to two or more resources by Stewart and Levin (1973). Dykhuizen and Dean (1994) reparametrized this model of scramble competition in terms more readily measured by experimentalists.

The fitness of strain 1 when rare $\left(w_{1 . \text { rare }}\right)$ is given by

$$
\begin{aligned}
W_{1 . \text { rare }} & =\frac{\mu_{1 . \text { rare }}}{\mu_{2 . \text { common }}} \\
& \approx W_{R 1} \frac{R_{o}}{R_{o}+S_{o}}+W_{S 1} \frac{S_{o}}{R_{o}+S_{o}},
\end{aligned}
$$

where $w_{R 1}=\mu_{R 1} / \mu_{R 2}$ and $w_{S 1}=\mu_{S 1} / \mu_{S 2}$ are the fitnesses on $100 \%$ on resource $R$ and $100 \%$ on resource $S$, respectively (Dykhuizen and Dean 1994). The fitness of a rare strain in the presence of both resources is simply the arithmetic mean of the fitnesses on the single resources, weighted by the relative abundance of the resources $\left(R_{o}\right.$ and $S_{o}$ ) in the fresh medium entering the chemostat growth chamber. Consequently, this resourcebased model of microbial competition has been entirely parameterized in terms of measurable fitnesses $\left(w_{1 . \text { rare }}, w_{R 1}\right.$, and $\left.w_{S 1}\right)$ and manipulable variables $\left(R_{o}\right.$ and $\left.S_{o}\right)$, and the measurable fitnesses have been parametrized in terms of the biochemical activity of the enzymes and equilibrium constant of the reaction. 
Microbial Experimental Evolution

Frequency-Dependent Selection

Inverting Equation 6 yields,

$$
\begin{aligned}
W_{2 . \text { common }}= & \frac{1}{W_{1 . \text { rare }}}=\frac{\mu_{2 . \text { common }}}{\mu_{1 . \text { rare }}} \\
\approx & \frac{1}{\frac{1}{W_{R 2}}\left[\frac{R_{o}}{R_{o}+S_{o}}\right]+\frac{1}{W_{S 2}}\left[\frac{S_{o}}{R_{o}+S_{o}}\right]},
\end{aligned}
$$

the fitness of strain 2 when "common" (and with $w_{R 1}=1 / w_{R 2}$ and $w_{S 1}=1 / w_{S 2}$ substituted). The competition model is symmetrical in terms of strains 1 and 2 and so the fitness of strain 1 when "common" can be now written out as (Lunzer et al. 2002):

$$
\begin{aligned}
W_{1 . \text { common }} & =\frac{\mu_{1 . \text { common }}}{\mu_{2 . \text { rare }}} \\
& \approx \frac{1}{\frac{1}{W_{R 1}}\left[\frac{R_{o}}{R_{o}+S_{o}}\right]+\frac{1}{W_{S 1}}\left[\frac{S_{o}}{R_{o}+S_{o}}\right]} .
\end{aligned}
$$

Equation 6 is the weighted arithmetic mean fitness of strain 1, but Equation 7 is the weighted harmonic mean fitness of strain 1. Harmonic means of positive numbers are less than arithmetic means. If a strain is fitter on one resource and less fit on the other there must exist a region on the resource axis where they both can coexist: where strain 1 is selectively favored when rare $\left(w_{1 . \text { rare }}>1\right)$ but selected against $\left(w_{1 . \text { common }}<\right.$ 1) when common.

These equations are based on the fact that in the chemostat, the concentrations of the two limiting nutrients are determined by the common strain. For the rare strain, the concentration of the preferred nutrient is high enough that its growth rate is higher than that of the common strain. Lunzer et al. (2002) tested this theory using strains TD2 and TD10, which were fitter lactulose and methylgalactose, respectively. Figure 5 shows the results. The lines are from the theory, not best-fit lines. The points are averages of four experiments. Once again, concepts in evolutionary biology can be tested experimentally.

\section{SURPRISES: TOP-DOWN STUDIES OF THE LACTOSE SYSTEM}

In the previous section, we started with a known system (specified genetic variation, a simple media, and a constant environment) and measured the initial selection coefficients over the first 80 to $100 \mathrm{~h}$ before any advantageous mutations rise to a high frequency in the culture. With this procedure, we can test quantitative theories of natural selection. However, with this procedure, we are testing the fitness differences of the genetic variation that we started with. Is this genetic variant the fittest one over the entire range of variation available? This question can be answered by starting a chemostat with a single strain and allowing the experiment to run for hundreds of generations as the bacteria adapt to the chemostat-media environment. The chemostats I use contain about $3 \times 10^{9}$ bacteria. If the per nucleotide mutation rate is about $10^{-10}$, then in just a few generations, every single nucleotide change possible will have been sampled. When this is performed and either individual strains or whole populations are sequenced, there are a bewildering array of mutations present (e.g., Zhong et al. 2004). However, there are some phenotypic changes that are regularly found. For example, there is strong selection for constitutivity of the pathway to metabolize the limiting sugar, be it maltose, which is positively regulated, lactose, fructose, raffinose-every sugar we have tried (D Guttman and DE Dykhuizen, unpubl.). Mutations in these regulatory genes are often seen, so sense can be made of this bewildering array of selected variation. The sorting out of what and why different mutations are selected in these long-term experiments will be a task for future generations of scientists.

\section{Evolution of Specialization}

Because we had shown that TD2 was a methylgalactose specialist and TD10 was a lactulose specialist (Lunzer et al. 2002), we decided that we would study the evolution of this balanced polymorphism (Dykhuizen and Dean 2004). Chemostats were started with a mixture of 28\% methylgalactoside and 72\% lactulose and the two specialists added. They converged to the 
D. Dykhuizen

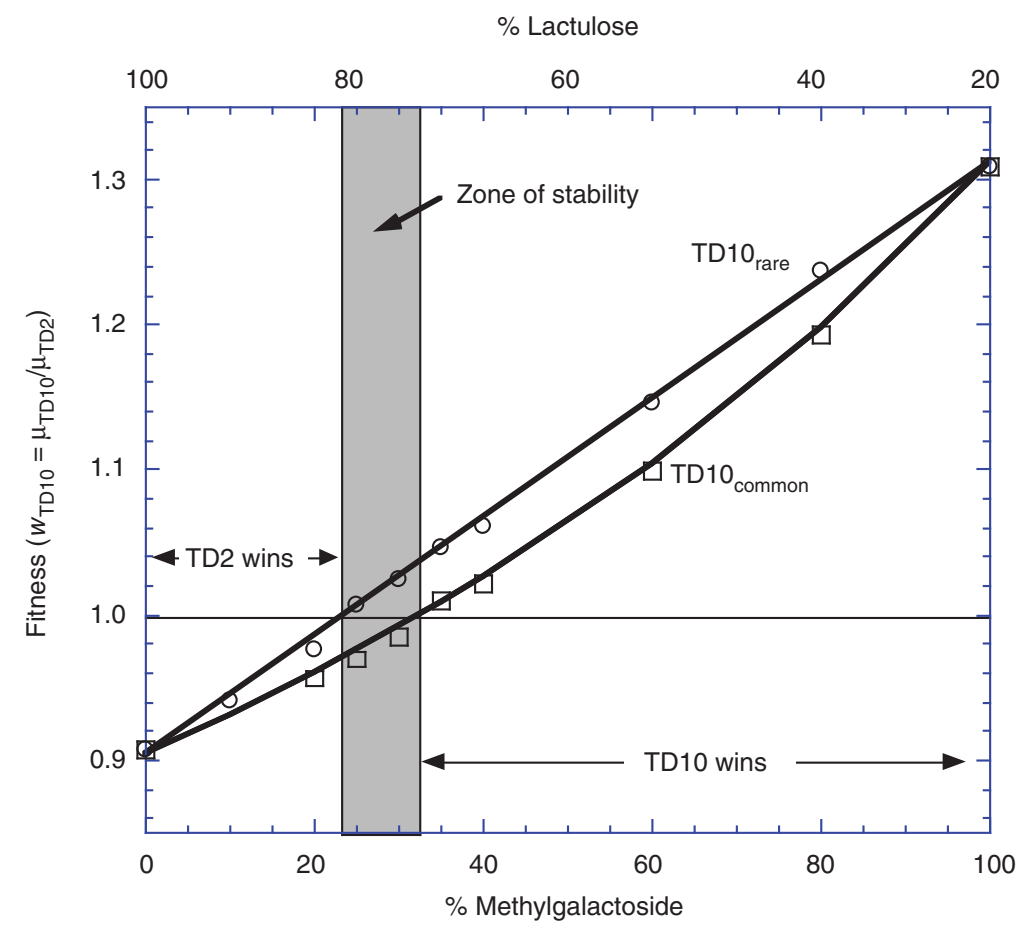

Figure 5. Effects of frequency in a complex environment. The solid lines are the theoretical fitnesses for TD2 and TD10 estimated using Equations 6 and 7. The points are the experimental points of the initial fitness measured at the various proportions of the galactosides. The gray strip is the region of coexistence. At $28 \%$ methylgalactoside and $72 \%$ lactulose the equilibrium is expected to be about $50-50$. When tested, this equilibrium was $62 \%$ TD 10 and $38 \%$ TD2.

predicted equilibrium (Lunzer et al. 2002) of $\sim 62 \%$ for the lactulose specialist. Over time, there was a lot of change in the frequencies of two strains. In five out of nine, separate chemostats fixed for one or the other strain within 200 generations. The other four remained polymorphic for 400-600 generations when the experiments were stopped. From these four chemostats, two strains were isolated from each chemostat, representing each of the specialists. When these strains were tested on $100 \%$ lactulose and 100\% methylgalactoside, it was often found that the strain that had been the lactulose specialist was now the methylgalactoside specialist and vice versa. Further work showed that the initial specialization did not matterother mutations were selected randomly to create lactulose specialists and methylgalactose specialists in this system.

Except maybe in one case, we never found generalists, in which the advantageous mutations were in the same clone. We always found specialists, the advantageous mutations in different clones. This could be because the generalists were less fit when both specialists were present or because this observation is an artifact of the mutation process (i.e., the probability the mutations creating the specializations were unlikely to happen in the same strain creating a generalist, but if they were combined it would result in an even fitter generalist). We did not determine this because the grant funding panels did not find this an interesting question and so we gave up pursuing it. However, it is clear that what happens when long-term experiments are run adds another level of complexity compared with the short-term experiments discussed above.

\section{Selection for Specialists}

To evolve specialists for different $\beta$-galactosides, I set up six chemostats. The media for two 
chemostats contained limiting lactose, two with limiting lactulose, and two with limiting galara. These chemostats were run for about 600 generations or just over 1200 hours. Single clones were isolated from each chemostat at the end of the run and genome sequenced. There were no genetic changes in the lactose operon in any of the strains, defeating the planned study of the evolution of specialization in either the permease or the $\beta$-galactosidase. However, there were amino acid changes in five of the six strains in $\mathrm{OmpF}$ (Table 1). This is not unexpected because the porin had a large control coefficient. All of the mutational changes decrease the size of the side chain on the amino acid and all are uncharged. This should decrease the steric and electrostatic interference of diffusion of $\beta$ galactosides through the pore, increasing flux through the pathway and increasing the growth rate. However, strains evolved on different $\beta$-galactosides have different numbers of these changes. The lactose-evolved strains have three changes, lactulose with two changes and gal-ara with one or none. Control coefficient for the porin gives a hint. It is 0.84 for lactose, 0.54 for lactulose, 0.58 for methylgalactoside, but the control coefficient of gal-ara was not determined (Dean 1995). Thus, maybe the selection for changes in the porin were stronger in lactose compared with the other two sugars.

The fitness of these six strains from the six chemostats was tested. The fitness of the two strains from the same limiting sugar is about the same, so fitnesses of pairs of strains were averaged. These are plotted in Figure 6. The lines $(\mathrm{A}, \mathrm{B}$, and $\mathrm{C})$ represent each pair of strains evolved and tested in the two environments. For example, line A represents the competition of lactulose-evolved strains against the lactoseevolved strains on lactose (left) and on lactulose (right). The other lines are constructed the same way. This figure shows specialization. Each strain is fitter in the environment in which it evolved than in the other environments. However, the lactose-evolved strains are fitter than the lactulose and gal-ara strains in all environments and the lactulose-evolved strains are fitter than the gal-ara strains in all environments. This order matches the number of changes in $o m p F$. If $o m p F$ is so important that the three changes in the lactose-evolved strains out competed the lactulose-evolved strains even on lactulose, why were not there more changes in the $o m p F$ of the lactulose-evolved strains? The same question applies to the gal-ara-evolved strains. Why are they selected against on gal-ara? Whatever is causing this strange phenomena cannot be simply chance. There must be some principle of natural selection here that we do not understand.

\section{THE IMPORTANCE OF CHANGING THE SELECTIVE ENVIRONMENT}

The next example shows that we still have a very primitive idea of the dynamics of natural selec-

Table 1. OmpF amino acid changes

\begin{tabular}{|c|c|c|c|c|c|}
\hline Strain & Sugar & AA change & Position & MW change & Nature of change \\
\hline \multirow[t]{3}{*}{ DD2457 } & Lactose & Arg $>$ Ser & 64 & -69 & $\mathrm{P}^{+}>\mathrm{PN}$ \\
\hline & & Asp $>$ Gly & 135 & -57 & $\mathrm{P}^{-}>\mathrm{NPN}$ \\
\hline & & Arg $>$ Cys & 154 & -53 & $\mathrm{P}^{+}>\mathrm{NPN}$ \\
\hline \multirow[t]{3}{*}{ DD2466 } & Lactose & Tyr $>$ Ser & 62 & -76 & $\mathrm{PN}>\mathrm{PN}$ \\
\hline & & Arg $>$ Pro & 104 & -59 & $\mathrm{P}^{+}>\mathrm{NPN}$ \\
\hline & & Asp $>$ Ala & 135 & -43 & $\mathrm{P}^{-}>\mathrm{NPN}$ \\
\hline \multirow[t]{2}{*}{ DD2459 } & Lactulose & Arg $>$ Pro & 104 & -76 & $\mathrm{P}^{+}>\mathrm{NPN}$ \\
\hline & & Glu $>$ Gly & 139 & -72 & $\mathrm{P}^{-}>\mathrm{NPN}$ \\
\hline \multirow[t]{2}{*}{ DD2467 } & Lactulose & $\operatorname{Arg}>$ His & 104 & -19 & $\mathrm{P}^{+}>\mathrm{PN}$ \\
\hline & & Glu $>$ Gly & 139 & -72 & $\mathrm{P}^{-}>\mathrm{NPN}$ \\
\hline DD2461 & gal-ara & None & & & \\
\hline DD2469 & gal-ara & Asp $>$ Gly & 135 & -57 & $\mathrm{P}^{-}>\mathrm{NPN}$ \\
\hline
\end{tabular}

P, Polar; NP, nonpolar; +, positive; -, negative; N, neutral. 


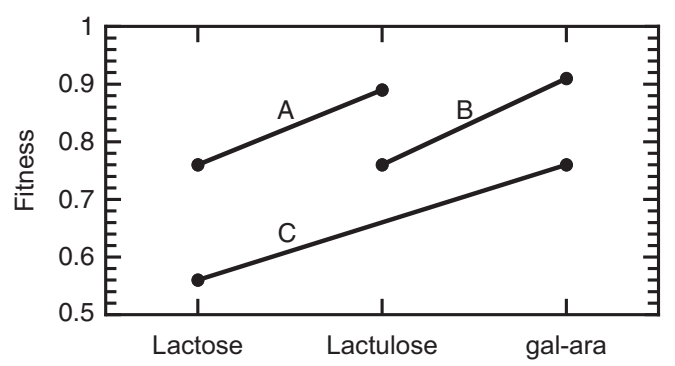

Figure 6. Fitness of strains evolved for 600 generations on the three limiting sugars. Line A, fitness of the lactulose-evolved strains compared with lactose-evolved strains. Line B, fitness of the gal-araevolved strains compared with the lactulose-evolved strains. Line C, fitness of the gal-ara-evolved strains compared with the lactose-evolved strains. Notice that the fitness of all these comparisons is less than one.

tion. Elena et al. (1998) randomly inserted an antibiotic-resistant marked derivative of transposon $\operatorname{Tn} 10$ into a single clone derived from a population isolated at generation 10,000 from a Lenski long-term line. The fitness of all independent 226 insertions was measured against the immediate ancestor, the clone isolated from a 10,000-generation population. Most insertions had little effect on fitness in the conditions that they had evolved in for 10,000 generations. In my experience, there is selection against an antibiotic resistance marker of a few percent. They found the average selection against the insertion lines of $3 \%$ and that includes the small minority of insertion lines that were very unfit. This alone is surprising-one would have expected a lot more selection because of gene inactivation caused by the transposon. Remold and Lenski (2001) used 26 randomly chosen strains out of the 226 insertion mutations from the Elena et al. (1998) experiment. This sample did not include any of the minority of insertion mutations that were very unfit. In these experiments, the strains were competed in a media in which the sugar provided was maltose rather than glucose. They found extensive fitness variation when the strains were competed in maltose but little when they were competed in glucose. Maltose is disaccharide of two glucose molecules. It is taken up into the cell by a different permease than glucose, cleaved into two glucose molecules, and then a phosphate group added to make glucose-6-phosphate. Glucose is phosphorylated as it is taken up into the cell to produce glucose-6-phosphate. All the rest of metabolism should be the same. Unless the insertion went into one of the few genes important in maltose metabolism, the fitness effects of the insertion mutations should be the same in glucose and maltose? Why are they so different? Changing the temperature of growth from $37^{\circ} \mathrm{C}$ to $28^{\circ} \mathrm{C}$ did not affect fitness (Remold and Lenski 2001). So, the explanation cannot be simply that the cells had evolved to grow only in a very narrow niche. Given this surprising result, it seems that our ideas about natural selection are incomplete at best and that a major effort is required, using experimental evolution, to create more satisfactory theories to explain natural selection.

\section{CONCLUSION}

Natural selection can be studied as a scientific concept using experimental evolution. But the complexity is immense. By holding the genetics and environment constant, we can test theories of natural selection. The robustness of these theories can then be tested varying the genetics (allowing new mutations into the population is the easiest because it is almost impossible not to have advantageous mutations arising to high frequencies if experiments are run for more than 100 generations) or by changing the environment.

\section{A Thought on Natural Selection}

I have not discussed the functional relationship between phenotype and fitness much. In the experiments using the lactose operon in E. coli, it was shown that increases in fitness were linearly related to increases in flux of the growth-limiting nutrient (Fig. 3). This is a special case because there is no change in the background that increased fitness. However, in the long-term experiments, in which advantageous mutations are incorporated into the population, the rateof-fitness increase decreases over time (Wiser 
et al. 2013). I propose that there is an optimal fitness given the environment. This has been an assumption in evolutionary biology for a long time because organisms are limited in their growth rate (i.e., their growth rate cannot become infinitely fast). I shall call the distance between the present fitness of the population and the optimum, the fitness space. An advantageous mutation in a population whose fitness is a long way from the optimum will have a much larger selection coefficient than the same mutation in a population whose fitness is much nearer to the optimum. It could be that the fitness of an advantageous mutation is a fixed proportion of the fitness space. Thus, fitness of an advantageous mutation will be a decreasing function of the increasing fitness of the population. This proposal has recently been experimentally verified. If two advantageous mu- tations are combined, the fitness would be less than expected (Fig. 7) and this is what is seen (Chou et al. 2011; Kahn et al. 2011). If two detrimental mutations are combined, the fitness loss is greater than expected (Fig. 8) and this has been seen (He et al. 2010). As the fitness of the population increases until it is near the optimum, selection coefficients of advantageous mutations would become so small that they would be effectively neutral (Hartl et al. 1985). But, in nature, the environment is always changing. How wide is the environment space that gives the same optimum? Changing from glucose to maltose changes the fitness of the background, thus involves a different optimum and presumably increases the fitness space to make the effects of the insertion mutations larger. A change in temperature does not seem to change the optimum.

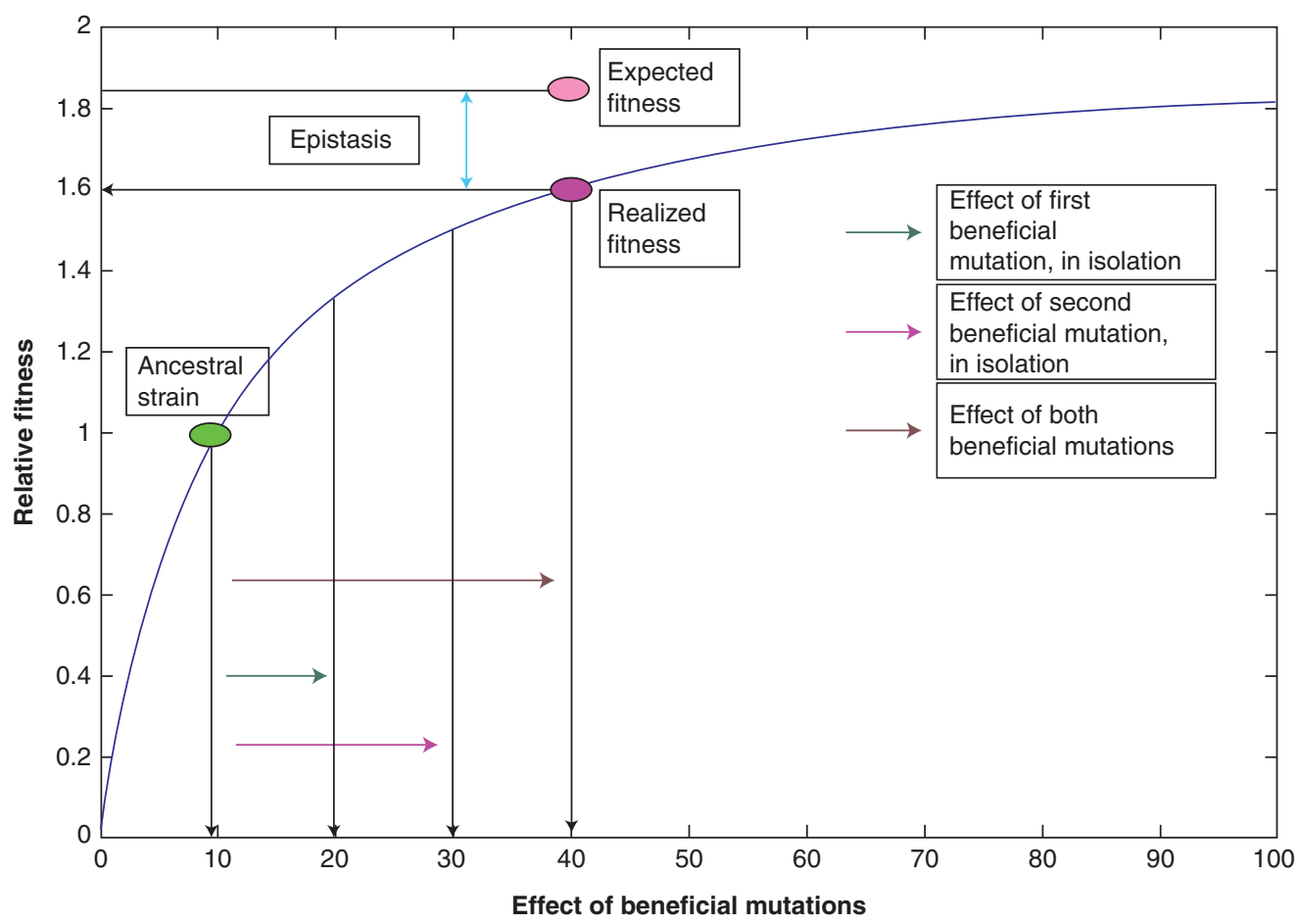

Figure 7. Negative epistasis for beneficial mutations. One advantageous mutation has an effect of 10 units and the other 20 units in a poorly adapted ancestor. The combination of the two advantageous mutations has an effect of 30 units and an expected fitness of over 1.8 compared with the ancestor. However, the measured fitness of the double mutations is only about 1.6, giving a negative epistasis because of the decrease in the fitness space. (Reproduced from a poster for the 2011 Microbial Population Biology Gordon Conference by the author along with Omar Warsi and Fabrizio Spagnolo.) 
D. Dykhuizen

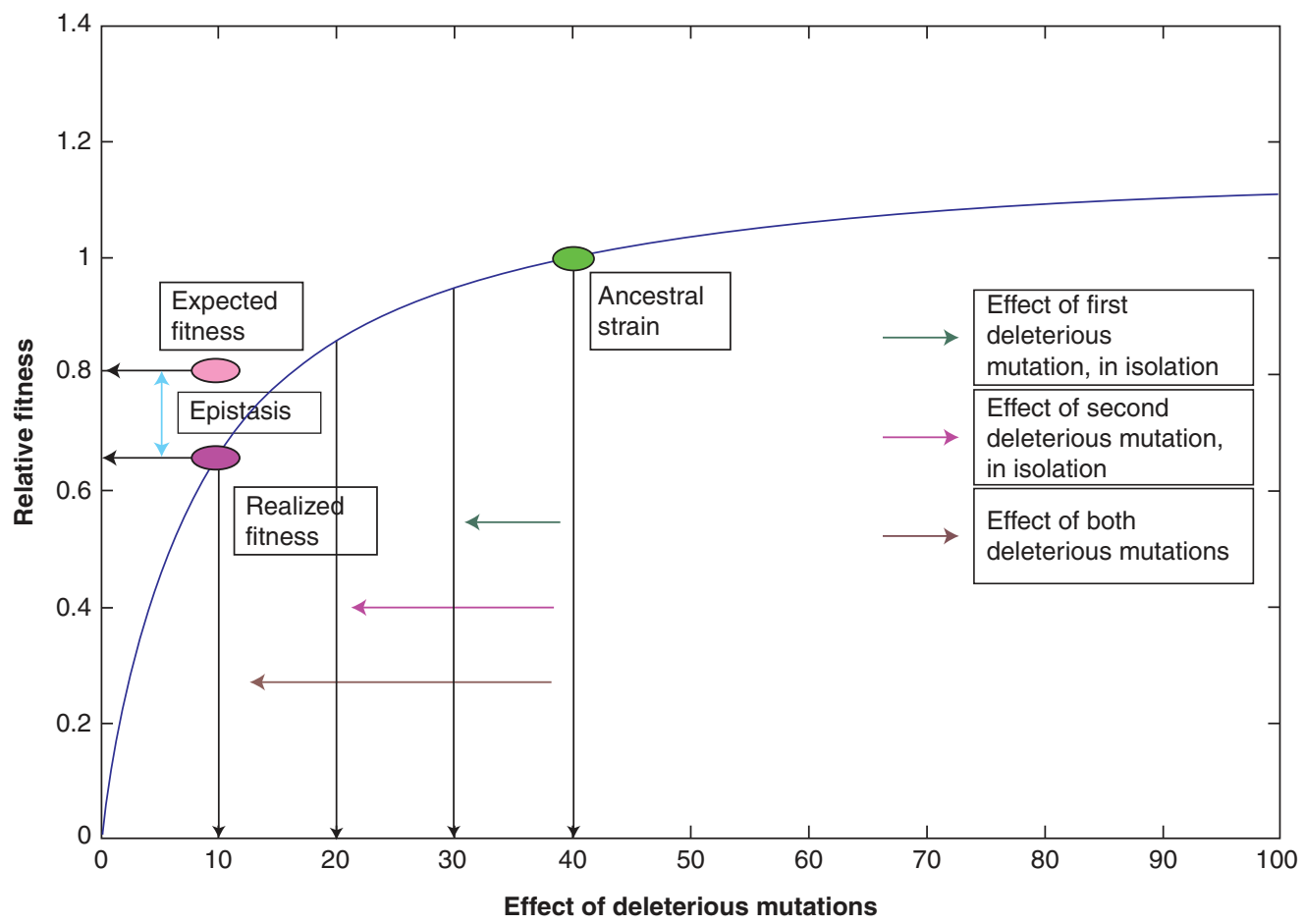

Figure 8. Negative epistasis for deleterious mutations. This figure is the logical opposite of Fig. 7. One detrimental mutant has an effect of 10 units and the other of 20 units. When combined this gives an effect of 30 units. When the fitnesses of the two independent detrimental mutations are combined the estimated fitness should be $\sim 0.8$. However, the measured fitness would be $\sim 0.65$. As above, the fitness is less than expected, so the epistasis is negative, but with an increase in the fitness space. (Reproduced from a poster for the 2011 Microbial Population Biology Gordon Conference by the author along with Omar Warsi and Fabrizio Spagnolo.)

\section{ACKNOWLEDGMENTS}

I thank Tony Dean, my collaborator on much of the work described here on the lactose operon. Without him, this work would never have been done.

\section{REFERENCES}

Atwood KC, Schneider LK, Ryan FJ. 1951. Periodic selection in Escherichia coli. Proc Natl Acad Sci 37: 146155 .

Chou HH, Chiu HC, Delaney NF, Serge D, Marx CJ. 2011. Diminishing returns epistasis among beneficial mutations decelerates adaptation. Science 332: 11901192.

Dean AM. 1989. Selection and neutrality at lactose operon in Escherichia coli. Genetics 123: 441-454.

Dean AM. 1995. A molecular investigation of genotype by environment interactions. Genetics 139: 19-33.

Dobzhansky T. 1951. Genetics and the origin of species, 3rd ed. Columbia University Press, New York.
Dykhuizen DE. 1993. Chemostats used for studying natural selection and adaptive evolution. Methods Enzymol 224: 613-631.

Dykhuizen DE. 1995. Natural selection and the single gene. In Population genetics of bacteria, 52nd symposium of the Society for General Microbiology (ed. Baumberg S, Young JPW, Wellington EMH, Saunders JR), pp. 161-173. Cambridge University Press, Cambridge.

Dykhuizen DE, Dean AM. 1990. Enzyme activity and fitness: Evolution in solution. Trends Ecol Evol 5: 257-262.

Dykhuizen DE, Dean AM. 1994. Predicted fitness changes along an environmental gradient. Evol Ecol 8: 1-18.

Dykhuizen DE, Dean AM. 2004. Evolution of specialists in an experimental microcosm. Genetics 167: 2015-2026.

Elena SF, Ekunwe L, Hajela N, Oden SA, Lenski RE. 1998. Distribution of fitness effects caused by random insertion mutations in Escherichia coli. Genetica 102/103: 349358.

Garland T, Rose MR. 2010. Experimental evolution: Concepts, methods, and applications of selection experiments. University of California Press, Berkeley CA.

Hall BG. 1984. The evolved $\beta$-galactosidase system of Escherichia coli. In Microorganisms as model systems for 
studying evolution (ed. Mortlock RP), pp. 165-185. Plenum, New York.

Hartl DL, Dykhuizen DE, Dean AM. 1985. Limits of adaptation: The evolution of selective neutrality. Genetics 111: 655-674.

He X, Qian W, Wang Z, Li Y, Zhang J. 2010. Prevalent positive epistasis in Escherichia coli and Saccharomyces cerevisiae metabolic networks. Nat Genet 42: 272-276.

Jacob F, Monod J. 1961. On the regulation of gene activity. Cold Spring Harbor Symp Quant Biol 26: 193-211.

Kacser H, Burns JA. 1973. The control of flux. Symp Soc Exp Biol 27: 65-104.

Kacser H, Burns JA. 1981. The molecular basis of dominance. Genetics 97: 639-666.

Kahn AI, Dinh DM, Schneider D, Lenski RE, Cooper TF 2011. Nagative epistasis between beneficial mutations in an evolving bacterial population. Science 332: 1192-1196.

Lunzer M, Natarajan A, Dykhuizen DE, Dean AM. 2002. Enzyme kinetics, substitutable resources and competition: From biochemistry to frequency dependent selection in lac. Genetics 162: 485-499.

Miller JH, Reznikoff WS. 1978. The Operon. Cold Spring Harbor Laboratory Press, Cold Spring Harbor, NY.

Monod J. 1942. Recherches sur la croissance des cultures bacteriennes. Hermann, Paris.

Monod J. 1950. La technique de culture continue. Theorie et applications. Ann Inst Pasteur Paris 79: 390-410.

Mortlock RP. 1984. Microorganisms as model systems for studying evolution. Plenum, New York.
Ochman H, Selander RK. 1984. Standard reference strains of Escherichia coli from natural populations. J Bacteriol 157: 690-693.

Ratcliff WC, Denison RF, Borrello M, Travisano M. 2012. Experimental evolution of multicellularity. Proc Natl Acad Sci 109: 1595-1600.

Remold SK, Lenski RE. 2001. Contribution of individual random mutations to genotype-by-environment interactions in Escherichia coli. Proc Natl Acad Sci 98: 1138811393.

Stewart FM, Levin BR. 1973. Partitioning of resources and the outcome of interspecific competition: A model and some general considerations. Am Nat 107: 171198.

Wiser MJ, Ribeck N, Lenski RE. 2013. Long term dynamics of adaptation in asexual populations. Science 342: $1364-$ 1367.

Woese CE. 1994. There must be a prokaryote somewhere: Microbiology in search for itself. Microbiol Rev 58: 1-9.

Zamenhof S, Eichhorn HH. 1967. Study of microbial evolution through loss of biosynthetic functions: Establishment of defective mutants. Nature 216: 456-458.

Zhang E, Ferenci T. 1999. OmpF changes and the complexity of Escherichia coli adaptation to prolonged lactose limitation. FEMS Microbiol Lett 176: 395-401.

Zhong S, Khodursky A, Dykhuizen DE, Dean AM. 2004. Evolutionary genomics of ecological specialization. Proc Natl Acad Sci 32 11719-11724. 


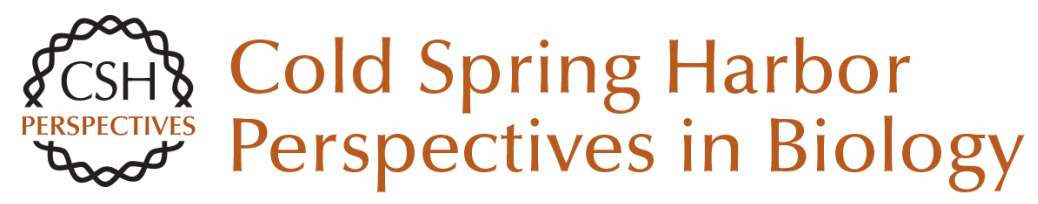

\title{
Thoughts Toward a Theory of Natural Selection: The Importance of Microbial Experimental Evolution
}

\author{
Daniel Dykhuizen
}

Cold Spring Harb Perspect Biol 2016; doi: 10.1101/cshperspect.a018044 originally published online January 8, 2016

\section{Subject Collection Microbial Evolution}

Not So Simple After All: Bacteria, Their Population Genetics, and Recombination William P. Hanage

Realizing Microbial Evolution Howard Ochman

Thoughts Toward a Theory of Natural Selection: The Importance of Microbial Experimental Evolution

Daniel Dykhuizen

Coevolution of the Organization and Structure of

Prokaryotic Genomes Marie Touchon and Eduardo P.C. Rocha

Mutation--The Engine of Evolution: Studying Mutation and Its Role in the Evolution of Bacteria Ruth Hershberg

The Origin of Mutants Under Selection: How Natural Selection Mimics Mutagenesis (Adaptive Mutation)

Sophie Maisnier-Patin and John R. Roth

Evolution of New Functions De Novo and from Preexisting Genes

Dan I. Andersson, Jon Jerlström-Hultqvist and Joakim Näsvall
Genome-Based Microbial Taxonomy Coming of Age

Philip Hugenholtz, Adam Skarshewski and Donovan H. Parks

Horizontal Gene Transfer and the History of Life Vincent Daubin and Gergely J. Szöllosi

Early Microbial Evolution: The Age of Anaerobes William F. Martin and Filipa L. Sousa

Microbial Speciation

B. Jesse Shapiro and Martin F. Polz

The Evolution of Campylobacter jejuni and Campylobacter coli

Samuel K. Sheppard and Martin C.J. Maiden

Paleobiological Perspectives on Early Microbial Evolution

Andrew H. Knoll

For additional articles in this collection, see http://cshperspectives.cshlp.org/cgi/collection/

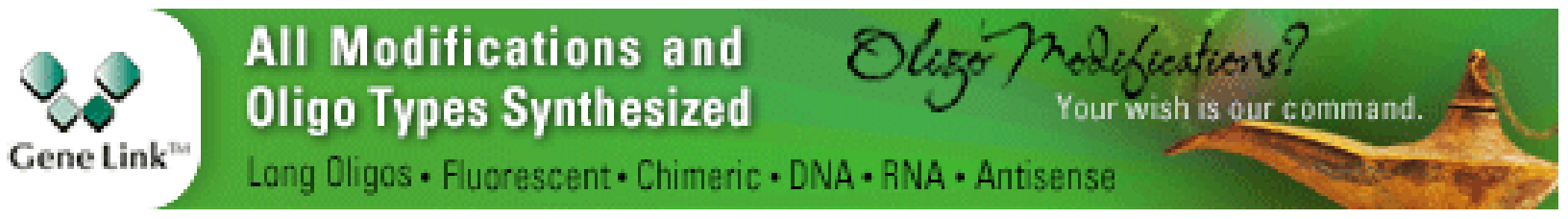

